\title{
Une approche du problème religieux dans la pensée de Michel Henry
}

\author{
Miguel Garcia-BARO \\ (Université Pontificale Comillas, Madrid)
}

\begin{abstract}
Phénoménologie de la vie et christianisme sont congruents au point que la réalité dont il s'agit en eux étant la même, leurs problèmes aussi sont les mêmes. ${ }^{1}$

Chaque crime est $l e$ crime $^{2}$
\end{abstract}

Nous devons une reconnaissance extraordinaire à Michel Henry. Il a mené à un point indépassable un programme philosophique d'une ambition inouïe depuis l'époque de Fichte. Ce programme a été tout inspiré par la méthode husserlienne de l'intuition. Une intuition d'ordre transcendantal, qui opère une réduction de l'ensemble des epistêmai qui encombrent, probablement à leur insu, la lumière de chair qu'est la vie subjective individuelle.

En parcourant ce chemin, les œuvres formidables de ces phénoménologues critiques de Husserl que furent notamment Heidegger et Merleau-Ponty, ont dû céder à l'élan originel de la méthode telle qu'elle fut premièrement conçue. Le Logos affectif, sous-jacent à toutes les modalités infinies de la vie en jouissance d'elle-même et toujours aussi en souffrance, parle vraiment la parole que ces autres penseurs octroyaient au Monde. Le Monde, tel que le décrit par exemple le jeune Levinas, ne parle jamais parce qu'il est mort, ou parce qu'il est l'horrible synthèse d'une vie insensée et de la mort. Les choses se taisent, mortes aussi, sauf à leur naissance dans notre peau et dans nos yeux. Le temps se résorbe dans l'éternité, pleine de sens, de vie. L'espace, à moins qu'il ne soit celui du corps et de sa limite, la Terre, devient la forme générale de l'oubli de soi, le commencement du mal.

Or l'altérité la plus extrême, celle qui sépare Dieu et l'homme et un homme d'un autre homme, se trouve entrer en conflit avec le programme de la phénoménologie radicale. Valables à jamais, la plupart des négations opérées par Henry n'en demeurent pas moins à mi-chemin de son œuvre ; mais l'amour, la liberté et le mal, tous ces phénomènes en rapport immédiat avec l'insoluble question du principium indi-

\footnotetext{
${ }^{1}$ M. Henry, «Le christianisme : une approche phénoménologique ? », dans Phénoménologie de la vie, tome IV. Sur l'éthique et la religion, Paris, PUF, « Epiméthée », 2004, p. 103.

${ }^{2}$ M. Henry, « Débat autour de l'œuvre de Michel Henry », dans ibid., p. 233.
} 
viduationis personae, exigent de nous certains écarts, aiguisés désormais par le regard lancé par Henry dans les profondeurs de la vie affective. Nous retournons ainsi, moyennant Henry, à des aperçus existentiels contenus dans le travail de Kierkegaard, et nous employons en partie les innovations méthodologiques apportées par Levinas à l'héritage de Husserl.

\section{1/ La religion comme forme de la culture et les fondements de la phénoménologie radicale}

Michel Henry, dans son article de 1985 intitulé « La question de la vie et de la culture dans la perspective d'une phénoménologie radicale », classe la religion parmi les formes supérieures de la culture. Cette phénoménologie radicale n'est que cette phénoménologie se refusant à se briser elle-même sur l'aporie qui a mis fin au radicalisme de Husserl : ne pas avoir pu «conférer un statut phénoménologique » ${ }^{1}$ à l'ultime instance responsable de toute phénoménalisation. En effet, si cette instance suprême est interprétée comme l'archi-impression constituant l'auto-temporalisation de la subjectivité, on voit simultanément dans cette Urimpression une fonction intentionnelle qui re-tient et pro-tient le flux du temps subjectif et se trouve elle-même plongée dans ce flux. Dès lors, c'est soit l'ek-stase du Temps qui incarne le rôle d'essence de la manifestation — et l'on se met à l'école de Bergson ; soit un acte ou pour mieux dire, l'action d'un ego impossible à « retenir » et ainsi à « réfléchir » — mais on réduit alors tacitement la distance qui sépare la phénoménologie de la tradition réflexive française, et l'on se met à l'école de Nabert. Or tout l'effort de la phénoménologie henryenne s'est concentré sur la conjuration de ce double risque qui, comme Charybde et Scylla, menace la possibilité d'une ontologie phénoménologique et d'une philosophie première de pure souche phénoménologique. Il s'agit, à l'évidence, de ne pas octroyer au temps lui-même le rôle de principe de phénoménalisation absolu et universel, et d'exiger du même coup de ce principe pré-temporel qu'il s'éprouve lui-même, s'auto-révèle sans aucun résidu d'obscurité, étranger à tout acte qui ne se possèderait pas soi-même et, peut-être, de manière à rendre possible un phénomène quasi-primordial du temps qui, à son tour, agira comme principe fondamental de la phénoménalisation du monde. Bien plus, cet acte prétemporel dont l'existence se doit de coïncider parfaitement, adéquatement, avec l'auto-révélation et avec l'ouverture de la possibilité de toute hétéro-révélation, ne doit à son tour ne pouvoir se décrire que comme une auto-affection : un se sentir soimême qui ne s'écoule jamais, qui n'est pas devenu, qui ne subit aucune altération susceptible de le temporaliser — un acte qui ne peut jamais finir.

L'on se demandera, tout d'abord, si les mots affection et sentir sont ici les plus appropriés. Ce dont il s'agit est de saisir à sa source la source de la vie, la vie se vivant

\footnotetext{
${ }^{1}$ Ibid., p. 17.
} 
elle-même avant de se livrer à quelque aventure au dehors de soi. Un des philosophes qui ont vu ou pressenti cette possibilité, au début du XI ${ }^{\text {ème }}$ siècle, Shlomo ben Gabirol, a cru apercevoir dans cette fontaine primordiale de la vie, de l'être et de la vérité, dans cet Un pré-temporel, c'est-à-dire éternel, la divine volonté — soit identifiée à Dieu lui-même, soit comme étant séparée d'une façon secrète, au-delà de toute connaissance, du Dieu énigmatique, inexprimable, absolument transcendant. Et il va sans dire que les mots affection et sentir ne sont pas les plus appropriés pour décrire un tel principe pré-mondain et phénoménologique du monde (dans les multiples sens que la philosophie néoplatonicienne avait su donner à ce terme devenu un peu plat dans la langue de bois philosophique moderne). Autopossession parfaite, autoposition qui est autoconnaissance, être qui s'identifie à la vérité, à l'éternité, à l'Unique : si l'être éternel est la vérité, il faut, en tout cas, lui donner le nom de Logos divin, lui attribuer la subjectivité, et ceci au sens le plus prégnant. Mais la question est de savoir si le terme de vie, que Michel Henry a toujours préféré, s'avère lui aussi vraiment adéquat. Poser cette question revient, c'est certain, à ébranler les fondements de la phénoménologie radicale, et un tel événement ne peut qu'être salué avec joie par ceux qui participent de cet élan qui nous conduit à éprouver les dernières chances et les derniers enjeux de ce que serait en vérité cette radicalisation extrême du phénoménologique. Et l'on conviendra d'ailleurs que c'est là seulement que devient sensée la prétention de nommer Dieu en phénoménologie

Or, dans la conférence par laquelle nous avons introduit ce cercle de problèmes, Henry propose une description particulièrement intéressante de ce qui à ses yeux justifie un tel emploi du terme de vie — en rapprochant le Principe phénoménologique de la Volonté de puissance nietzschéenne, c'est-à-dire de la divinité (qu'elle reçoive le nom de Dionysos ou le nom de Meqor Hayyim importe relativement peu).

Un premier moment de la description nous conduit droit à la finitude, à l'expérience de celui qui qui ne saurait transférer au divin (quel qu'il soit, fût-il polythéiste) les phénomènes qu'il repère comme essentiels à l'autorévélation de la vie, et ceci bien qu'ils autorisent à parler de corps subjectif en un sens biranien radicalisé : «Le savoir qui a rendu possible le mouvement des mains, celui des yeux, l'acte de se lever, de gravir l'escalier, de boire et de manger, le repos lui-même, est le savoir de la vie ». Il s'agit ici de noter une différence capitale entre gravir un escalier, boire, manger ou se reposer, et le savoir qui permet ces actes - savoir corporel, charnel, qui accompagne ces actions subjectives et corporelles en les éprouvant d'une façon telle que, simultanément, il ouvre leur possibilité même. Ce savoir pré-temporel qui reste ici sous-jacent est la chair vivante, la vie, ma vie sensible, première par rapport aux autres savoirs qui me sont aussi accessibles et qui relèveraient de ce que Henry nomme ici le savoir scientifique et le savoir de la conscience (ce dernier étant le savoir conceptuel le plus proche du savoir sensible ou

\footnotetext{
${ }^{1}$ Ibid., p. 18.
} 
de la vie). Un tel savoir sensible peut-il dès lors se trouver transféré à vie divine ? Ne nous hâtons pas de répondre.

Un second moment de la description permet de faire un pas de plus. Elle prolonge la définition de la «culture » comme autotransformation de la vie, « le mouvement par lequel elle ne cesse de se modifier soi-même afin de parvenir à des formes de réalisation et d'accomplissement plus hautes, afin de s'accroître $» .{ }^{1}$ De toute évidence, une telle affirmation suppose que la vie ait des potentialités, voire que ces potentialités la définissent dès lors que cet accroissement — d'abord saisi au moyen du concept de culture - appartient à son essence et n'obéit pas à un élan qu'elle devrait recevoir du dehors. Et de fait, le savoir sensible ou savoir de la vie reçoit chez Henry le nom classique de " praxis » (en un sens phénoménologique radical bien sûr), de sorte que praxis et culture ne soient plus que deux aspects de l'essence même de la vie, identifiés l'un à l'autre dans le regard du philosophe. ${ }^{2}$ L'éternelle venue de la vie en elle-même ne se restreint point à être une répétition monotone, dépourvue de tout sens, insupportable, du Même. Ce Même devient soi-même par une sorte d'auto-accroissement essentiel, que l'on pourra étroitement rapprocher des descriptions bergsoniennes de la durée réelle en tant que subjective, et ceci en dépit des déclarations expresses de Henry que beaucoup parmi nous avons entendu de sa bouche avec plus d'emphase que l'on n'en retrouve dans ses textes. Et si cette « ivresse » de la vie comme telle admet, au dire de Henry, une forme immédiate de praxis ou de culture (correspondant au niveau des besoins vitaux), c'est le travail qui l'incarne, en tant qu'il ne vise pas à annuler les besoins mais justement à les accroître comme tels dans la mesure où, momentanément satisfaits, ils promettent de renaître avec d'autant plus de puissance. En ce sens, l'art, la morale et la religion ainsi que la philosophie radicale elle-même — constituent les formes «supérieures » de la culture, de la praxis. ${ }^{3}$

\section{2/ La vie comme amour, dans l'ambiguïté du Fini et de l'Absolu}

Or cette supériorité ne désigne pas, ne peut pas désigner un saut, le dépassement d'une limite déterminée. Qui pourrait imposer une injonction quelconque à la vie, si tout ce qui gît au dehors de la vie est mort (ou chose) ? Le besoin sensible doit se prolonger dans un besoin supérieur : le besoin de manger, en art de la cuisine et en raffinement gourmet; celui de s'abriter en édifice de style Bauhaus (l'exemple est

\footnotetext{
${ }^{1}$ Ibid., p. 19 sqq.

${ }^{2}$ Bien sûr, l'orthodoxie henryenne exige que nous considérions la vie comme ce qui produit constamment sa propre essence, et non pas l'auto-accroissement comme ce qui dériverait d'une loi immanente contenue dans une certaine essence stable de la vie. Cf. sur ce point ibid., p. 104.

${ }^{3}$ Une religion, «c'est un ensemble de pratiques ». «La religion étant le lien intérieur qui unit l'homme à Dieu, les pratiques religieuses consistent dans l'actualisation phénoménologique de ce lien. Dans cette union vécue avec Dieu, la vie du fidèle est transformée » (ibid., p. 113).
} 
de Henry lui-même) ; le cri de la chair en religion. ${ }^{1}$ Mais la religion est adoration. La vie qui adore peut-elle être la vie elle-même ? Ce serait, bien sûr, la forme la plus développée du travail (avodah, cultus) : l'éclosion du besoin dans son essence la plus pure, déjà indépassable, de besoin.

À l'extrême opposé du sentiment de l'être comme fatigue et nausée qui font jaillir le désir d'évasion comme première esquisse de la religion, cette approche si nietzschéenne de la nature de la religion condamne toute évasion de ce style lévinassien - comme le principe même de la barbarie : telle serait en effet l'origine de la faiblesse, ce mystère d'une auto-divergence de la vie en raison de la possibilité étonnante et effrayante que « la force infinie de la vie se tourne contre soi — dans la mauvaise conscience, la haine, le ressentiment. $»^{2}$ La religion ainsi saisie connaît le sacré de la vie, mais ne le confond-elle pas avec le saint ? La position de Henry implique ici que nulle différence ne puisse s'établir entre le sacré et le saint, et que Dieu nécessite bien l'être, puisque c'est Son essence même - le blasphème étant alors de réclamer une pensée de Dieu sans l'être. L'héroïsme de la vie, d'être fidèle à son auto-accroissement, devient - comme tout hérö̈sme, mais celui-ci en est la forme centrale — presque insupportable, et ainsi la vie folle, égarée, se trouve désormais portée par un élan absurde : se libérer de soi-même, « se défaire de soi », s'affirmer vigoureusement en se donnant l'illusion de se rejeter. Du reste, il est évident que, parmi les besoins de la vie, ne figure pas la croissance de la lucidité sur elle-même. Ainsi cette Atlantide qu'était la ville d'Aliahova s'enfonce-t-elle sous l'emprise des hommes dégoûtés de l'excès de vie — qui, manifestement, comporte toutes sortes d'accroissements des besoins, sauf celui de la vérité. ${ }^{3}$

Mais l'aspect le plus inquiétant de cette première saisie de la religion réside dans l'indistinction entre la vie absolue et la vie finie à laquelle elle semble condamnée. Et c'est, à mon sens, un conflit perpétuel dans la pensée henryenne.

On le voit clairement, chaque description de l'essence de la vie fournie par Henry contient le mot clé de pathos. Susceptible de culture, la vie l'est-elle en elle-même ou seulement en tant que vie finie ? Potentialités qui s'actualisent, joie de soi qui s'accroît toujours en éprouvant toujours à neuf ce qui, au commencement, était enveloppé en soi-même, s'épanouissant en autant d'occasions de jouissance au fur et à mesure qu'elle jaillit de son fond obscur — une telle vie n'est-elle pas essentiellement une vie finie, même si la pensée de la noesis noeseos nous semble, dans notre

\footnotetext{
${ }^{1}$ L'art est l'« éthique de la sensibilité, développement et intensification de tous ses pouvoirs jusqu'à ce point où leur exercice exalté se change en ivresse de l'expérience esthétique » (ER 36, 1986). Plus intéressante et juste encore me semble cette autre affirmation : «L'art réintroduit ce que la science galiléenne avait mis entre parenthèses » (ibid., p. 50).

${ }^{2}$ Ibid., p. 23.

${ }^{3}$ Je me permets de renvoyer à mon interprétation du roman henryen L'amour les yeux fermés: "The black river that feeds all. A piece of literature expanding Michel Henry's philosophy », dans J. Hanson \& M. R. KELLY (éds.), Michel Henry and the Affects of Thought, Londres, Continuum, " Continuum Studies in Continental Philosophy ».
} 
naïveté, exiger quelque chose d'une évolution créatrice, et que l'éternité nous offre le visage d'une durée infiniment concentrée et puissante ?

Certes, ce sont certainement des anthropomorphismes, et les déclarations de Henry sont formelles : «dans ce s'éprouver soi-même, la vie fait l'épreuve de soi comme de ce qu'elle n'a pas posé elle-même mais qui lui advient et ne cesse de lui advenir - comme de quelque chose qu'elle subit constamment dans un subir plus fort que sa liberté ». Personne n'a pas pu poser cette richesse inconnue conduite à se développer, exceptée la vie absolue - et ceci dans le tréfonds de la vie finie. Mais cette autre déclaration, sur la même page, est tout aussi formelle : cet advenir en soi est « perpétuel» et « éternel », ce subir consiste dans l'épreuve du fait que « la vie ne cesse d'être assaillie par son être propre et submergée par lui », lequel « la jette en avant, la pousse à 1 'action $»{ }^{1}$ Or ces paroles ne sont applicables qu'à la vie infinie. Si nous osions, au point où nous en sommes, la nommer Dieu, il faudrait dire que Dieu n'est ni libre ni personnel, et que son être obscur, en soi, demande une autothéophanie qui antécède en lui-même la liberté et, bien sûr, la personnalité. Quelque chose comme la substance de la divinité (ousía) dominerait alors sa personnalité (l'hypostase du Père, dans le langage de la théologie des Pères grecs, magnifiquement réexposée, parmi tant d'autres contemporains, par Ioannis Zizioulas). ${ }^{2}$ Et la liberté deviendrait, en Dieu et dans la vie finie, une erreur de perspective, une erreur philosophique. Si tant est qu'il y en ait, la liberté resterait réduite à un phénomène du monde, à la subjectivité appréhendée comme mondaine ou empirique. Rien au champ transcendantal ne serait libre.

Dès lors la question se pose : pourquoi établir une éthique, formuler la proposition subjective d'une morale ? Pourquoi même une réflexion morale ? Ces phénomènes ne pourraient appartenir qu'à la vie finie, bien entendu, comprise sous l'angle de son étonnante difficulté à «se représenter ce qu'elle veut ${ }^{3}{ }^{3}$ Certes, ce qu'elle veut répond à une téléologie immanente susceptible, néanmoins, de s'égarer et de s'oublier (en vertu, sans doute, de quelque particularité elle-même contenue dans l'essence de la vie - finie —, car rien ne peut provenir des choses mortes). Mais immédiatement, Henry répète ce mouvement par lequel il estompe presque totalement la distance entre Vie absolue et vie finie - comme s'il s'agissait de deux hypostases de la même vie. Et la seule justification d'un tel geste résiderait dans la reconnaissance d'un Désir constitutif du noyau même de la vie, en vertu duquel l'épreuve de soi-même signifierait toujours, et pour tout type de vie, une souffrance immanente. ${ }^{4}$ La valeur absolue, qui est la vie, enferme dans son secret, à notre surprise, une multiplicité de potentialités ou «caractères ontologiques », chacun vou-

\footnotetext{
${ }^{1}$ M. Henry, Phénoménologie de la vie, tome IV, op. cit., p. 21.

${ }^{2} \mathrm{Cf}$. le premier chapitre de son excellent livre L'Être ecclésial, Paris, Labor et Fides, 1981.

${ }^{3}$ M. Henry, Phénoménologie de la vie, tome IV, op. cit., ibid., p. 33.

${ }^{4}$ Cf. ibid.
} 
lant «sa plus haute réalisation ». ${ }^{1}$ Confronté au temps mort du monde et de ses choses, la floraison de ces pouvoirs de la vie exigerait alors un « historial». Et chaque phase de cet historial prétemporel mais architemporel s'articulerait en deux moments : un "se souffrir» qui, culminant dans un «se jouir», dériverait de l'épreuve de l'élan par lequel le pathos se dépasserait toujours vers la nouvelle phase d'un nouveau pathos (à son tour dépassé par l'auto-accroissement dans l'autojouissance). ${ }^{2}$ Or cette auto-jouissance est bien qualifiée, dans les développements postérieurs de cette saisie essentielle, comme amour. Un amour sans altérité : l'amour de la vie pour elle-même. ${ }^{3}$ Cherchons la méthode pour progresser dans le champ de ces problèmes qui de tous côtés nous assaillent.

\section{3/ La Terre, le Soi, le Je et le Retournement}

Le premier geste — bien déroutant ! - , serait de revenir aux positions originelles de Henry, très proches de Maine de Biran et défendues uniquement dans l'essai dédié à ce penseur, et d'admettre que le «corps organique» n'est pas une chose morte — bien qu'il diffère du « corps originel », c'est-à-dire de la vie ipséisée finie en tant qu'ensemble de ses potentialités - mais une limite extérieure-intérieure de ce corps originel : ce qui cède à son effort. Et de fait, la description du déploiement de nos potentialités comme effort exige cette limite, non pleinement extérieure, qui cède. Mais cette limite, à son tour, ne pourrait opposer de résistance relative au pouvoir originel de la vie si, en dernière instance, il n'achoppait lui-même sur une limite plus proprement extérieure, et pour sa part incapable de céder : la « ligne de résistance absolue de la Terre », qui ne tire néanmoins son existence que d'être la limite pratique du corps (dans les deux sens du terme). ${ }^{4}$ Vie-corps et Terre se co-proprient ainsi, ou se corps-proprient. Est-ce la Vie absolue qui exige la Terre ? L'on pourra en douter.

Un second élément nous est fourni par quelques précisions apportées par Henry à ce qui constitue à ses yeux le thème propre de la religion : « le lien intérieur qui relie chaque vivant à la vie [...]. [Ce] lien a son site dans la vie, il est vécu de diverses façons, toutes prescrites cependant par l'essence originaire de la vie et le procès selon lequel elle génère en elle le vivant $\gg{ }^{5}$ Évidemment, si l'on admet ce point de vue, on reconnaît par là même que morale et éthique dépendent toutes deux de la religion.

\footnotetext{
${ }^{1}$ Ibid., p. 35 et p. 34.

${ }^{2}$ En certains endroits, Henry va même jusqu'à parler de la « temporalité immanente de la Vie », qui n'est, bien sûr, que ce passage de la souffrance dans la joie et de la joie dans la souffrance que je viens de décrire. Cf. par exemple ibid., p. 92.

${ }^{3}$ Cf. par exemple ibid., p. 63.

${ }^{4}$ Ibid., p. 37.

${ }^{5}$ Ibid., p. 54.
} 
Mais l'ambiguïté de la finitude et de l'infini persiste, car il y a un Vivant qui est essentiellement premier : le Logos charnel dans lequel la Vie absolue s'auto-révèle en s'ipséisant. Le sujet premier et exemplaire de la religion et de la morale est cet Archi-Fils de la Vie auquel se rapporte la génération du reste des vivants, fils dans le Fils. C'est du vivant fini que la constitution du monde relève, et non pas de l'ArchiFils. ${ }^{1}$ Mais nous ignorons tout d'abord si la Terre appartient, au moins par un aspect de sa réalité, au Monde. Il est clair que le Monde ne peut jamais entrer dans la corpspropriation, car il n'est composé que de choses mortes et vaut lui-même comme figure universelle ou formelle de la Mort; mais si la Terre diffère du corps subjectif — et comment ne pas le reconnaître? —, d'où peut-elle bien procéder? Est-ce justement le Logos qui constitue la Terre comme une portion inévitable de l'essence du vivant fini qu'il génère ?

Tout ego, sauf l'Archi-Fils, naît et, en ce sens, doit passer du nominatif auquel le cantonne la philosophie transcendantale idéaliste, à l'accusatif où le localise la phénoménologie radicale. Mais cette naissance est une naissance transcendantale, ce qui signifie qu'elle n'est dans son principe «ni ponctuelle ni événementielle », mais plutôt une « condition que rien ne peut interrompre ${ }^{2}{ }^{2}$ Or si cette condition est ellemême phénoménologique, il faudra, par exemple, la retrouver comme un soubassement auto-donné de mon ego. Henry a tenté d'exprimer cette articulation dans une formule qui, à l'évidence, rejoint les plus célèbres audaces d'Eckhart : le soi de mon ego (l'accusatif précédant mon nominatif habituel) est l'Ipséité « originelle cogénérée dans l'auto-génération de la vie ${ }^{3}{ }^{3}$ Ou pour le dire autrement, mon soi, fondement de mon ego, coïncide avec le Logos de la Vie. Et en ceci, il se doit sans doute de coïncider avec tout « autre » Soi dans tout autre vivant fini et, en général, dans tout vivant. Dès lors, il y aura une pluralité infinie d'egos individuels, mais tous soutenus dans l'identité d'un Soi qui n'est que le Logos charnel du Père. Moi, je peux toutes les potentialités mises d'abord dans «mon » soi, dans le soi. ${ }^{4}$ Le problème s'est ainsi déplacé : ce qu'il s'agit de saisir, c'est l'entrée en possession de ces pouvoirs qui sera, si l'on parle avec rigueur, la naissance, ma naissance.

Henry a voulu décrire d'un seul mot ce « passage » dont tout dépend : « retournement $»{ }^{5}$ Mais l'on ne saisit alors que l'effet du processus caché, au terme duquel je, le moi, considère mes pouvoirs comme miens, me prends pour leur source absolue, les exerce librement, c'est-à-dire en seul maître. La vérité est que j'en ai été investi en recevant mon soi (mon moi avant le retournement en je), mais que je suis mis d'emblée dans la possibilité immédiate d'oublier ma source, ma permanente condi-

\footnotetext{
${ }^{1}$ Cf. ibid., p. 55.

${ }^{2}$ Ibid., p. 56.

${ }^{3}$ Ce point essentiel se manifeste avec une grande clarté dans la contribution henryenne à l'Archivio di Filosofia (1996); cf. ibid., p. 57.

${ }^{4}$ Le Premier Vivant, qui porte aussi le nom de Soi transcendantal, constitue, en effet, « l'essence de 1'homme et ainsi de tout Individu au sens humain » (ibid., p. 107-108).

${ }^{5}$ Ibid., p. 58.
} 
tion d'existence, mon intimité. Voilà le Maître intérieur traduit dans les termes d'une immanence extraordinairement audacieuse, parce qu'il n'est pas la Vérité illuminant sur l'œil de mon esprit les vérités éternelles, mais le Vivant qui concentre en Lui tous les pouvoirs de tous les vivants, à leur source, non encore explicités en ensembles individuels de pouvoirs égoïques, non encore éparpillés - comme le Noûs conditionne la troisième hypostase plotinienne : l'Âme.

Dans cette perspective, qui exige encore des approfondissements ontologique très serrés, éthique et religion sont saisies en leur essence bien plus complètement que dans notre approche antérieure, et en une proximité évidente avec l'augustinisme : le but de l'éthique est la seconde naissance, c'est-à-dire « la restauration du lien religieux » explicite. ${ }^{1}$ Il s'agit bien de retourner le retournement, de délaisser l'appropriation initiale signifiée par le mot « je », de la conversio d'une perversio originelle. C'est en moi-même, et non plus sur l'acies mentis, que ce drame historial se joue : la vie divine ipséisée demeure au-dessous de ma chair qui pèche en naissant et qui se sauve en renaissant. ${ }^{2}$ C'est au-dedans de moi-même que le Logos est rejeté par la chair finie - on dirait plus volontiers par l'Esprit, mais la théologie henryenne, sauf dans des passages assez forcés, contient plutôt une Dualité qu'une Trinité. $^{3}$

\section{4/ La souffrance et le mal moral}

Un des mystères liés à l'interprétation globale de la phénoménologie radicale de Michel Henry est celui de la compréhension juste du Monde des Choses, du Monde de la Mort. S'agit-il d'un second principe (ou des conséquences d'un second principe) qui s'opposerait à la Vie, soit à la source de toute existence réelle (voire à l'existence réelle elle-même) ? Un principe propre au Monde de la Mort toutefois, indépendant de la Vie, paraît immédiatement impossible, car il devrait vivre en quelque façon, si doit justement en dériver un le Monde. Et si l'on insiste sur l'unicité de la Vie comme réalité et source de réalité, forte est la tentation de saisir la Mort comme étant, justement, de la Vie aliénée, terminée, épuisée, grâce à un dispositif lui-même vivant — et ainsi de soustraire la vie à un domaine demeurant pourtant essentiellement vital. Or c'est sans doute la seule manière cohérente de comprendre l'intention de Henry, si difficile qu'il soit dès lors d'expliciter cette cohérence sans violenter les règles de la pensée comme pensée vivante.

\footnotetext{
${ }^{1}$ Ibid., p. 60.

${ }^{2}$ L'éthique elle-même « n'est plus rien d'autre que la manière dont le vivant vit sa naissance transcendantale de telle façon qu'il puisse la revivre» (ibid., p. 109). Et p. 111: «Le mouvement de cette auto-transformation est l'éthique, son site est la religion ».

${ }^{3}$ En tout cas, si ipse et personne s'équivalent, ce ne sera pas le Père (la Vie absolue) qui pourra être compris comme hypostase ou personne, mais seulement le Fils. La Vie engendre l'Ipse dans la pure nécessité de son procès éternel.
} 
Un témoignage tranche sans ambiguïté en faveur de ce monisme ultime de la Vie, dans la contribution de Henry au colloque sur l'argument ontologique tenu à Rome en 1990. Posant la thèse initiale selon laquelle toute existence réelle tient sa réalité de l'intériorité radicale qu'est l'auto-affection ou la Vie, il n'en affirme pas moins que, de cette présentation première ou de cette révélation immanente, une représentation demeure possible « dans la pensée », en vertu de laquelle « l'existence réelle devient son contraire : une irréalité noématique ». Et comme c'est déjà le cas pour la plupart des intuitions (au sens husserlien des Recherches logiques par exemple), ce procès par lequel l'être se vide de sa vie et de son propre être s'achèverait dans l'instance linguistique ou " symbolique ». ${ }^{1}$ L'intentionnalité dite intuitive dé-réaliserait ce qui tout d'abord était bien réel. Le but de ce rappel essentiel est alors de s'opposer radicalement à la possibilité de reconnaître quelque valeur que ce soit à la preuve anselmienne de l'existence de Dieu (ou plutôt à la version de cette preuve que certains logiciens américains - Kalish and Montague - qualifient très justement de «blasphématoirement breve »). La théologie ne serait alors possible que comme phénoménologie (radicale), à supposer que le langage théologique fonctionne sur le fondement d'une épreuve immanente de Dieu. Dieu aurait alors à se révéler non point dans la foi d'un autre ou dans la signifiance d'un récit, mais dans la chair subjective de la vie, de ma vie, et comme faisant partie de ce qui, en général, mérite d'abord le titre d'auto-affection. Évidemment, il y a là de quoi effrayer des théologiens orthodoxes.

Mais avant de pénétrer, autant que faire se peut, cette expérience charnelle de Dieu, ${ }^{2}$ l'on fera bien de souligner que, habituellement, le mot être s'applique au Monde et aux corrélats noématiques de l'intuition, et que c'est en se guidant sur la super-objectivité de certains d'entre eux — les idées, les essences — que l'on saisit couramment l'éternité : le manque de Temps Mondial s'interprète alors comme le noyau réel de l'éternité, là où il s'agirait davantage de comprendre que « la vie n'est pas » mais « advient à soi en soi » ${ }^{3}$, comme un don qu'elle se fait à elle-même sous l'espèce d'une suprême Bonté. Aussi serions-nous encouragés à distinguer plus proprement l'Historial et le Dimensional - le premier comme intérieur à Dieu, et le second, en tant qu'il prend sa source dans la projection intentionnelle du Temps Cosmique, comme la forme même de la Mort. ${ }^{4}$ Mais ce n'est pas Dieu seul qui habite l'Historial. Il y a encore le Mal, le mal réel et non point le mal fantasmatique que personne n'éprouve. Le Mal a-t-il quelque chose à voir avec cet élément caché dans la vie dont procèderait la Mort ? Ou bien cet élément ne contient-il rien de plus

\footnotetext{
${ }^{1}$ Cf. ibid., p. 79 : « Dans la chair de la vie il n’y a place pour aucune irréalité noématique ».

${ }^{2} \mathrm{Cf}$. cette expression ahurissante: ... « La pro-jection noématique de ce qui se trouve d'ores et déjà édifié intérieurement comme sensation » (ibid., p. 88 ; c'est moi qui souligne).

${ }^{3}$ Ibid., p. 76. Cf. aussi p. 120 ; « La vie comprise en son sens originel n'est ni un étant, ni un être, ni l'Être, mais un procès »; p. 161, Henry parle d'un « devenir (le contraire d'une "substance" ou d'une "chose") ». D'ailleurs, « la vie est tout sauf une tautologie » (ibid., p. 148).

${ }^{4}$ Cf. ibid., p. 218 : « Je n'ai jamais employé le mot temps à propos du procès interne de la vie ».
} 
que la pensée (intentionnelle) et le langage — des éléments en apparence plutôt innocents et qui, à la différence du Mal, ne semblent pas pouvoir être si diamétralement opposés à Dieu?

Il faut ici reconnaître que tout Monde est essentiellement contingent, alors que la Vie est éternelle et, par conséquent, le Nécessaire comme tel. Cette contingence est attribuée d'abord à la re-présentation de ce Monde-ci parmi tant d'autres possibles. Quant à la fonction objectivante, "vidante », de la pensée intentionnelle, est-elle aussi nécessaire que la vie elle-même ? Peut-être se lie-t-elle inévitablement à la présence du Mal, comme nous l'avons suggéré ${ }^{1}$ et, en ce cas, le problème éthique et religieux se formulera comme celui de la contingence ou de la nécessité du Mal. Aurait-il été possible de toujours penser sans intentionnalité, comme c'est le cas, selon Henry, dans l'éthique, l'art et la religion?

Nous savons déjà que nous souffrons, fondamentalement, lorsque naît en nous le vouloir impuissant de nous défaire de nous-mêmes, et lorsque la vie ne poursuit plus son mouvement historial d'auto-accroissement. Mais si l'on soutenait que c'est proprement aussi souffrir le Mal, cet aspect de tout pathos qui, nous l'avons vu, consiste en un recevoir ou un se voir affecté en conflit avec la force de l'élan immanent de la vie, on confondrait absolument le mal véritable, c'est-à-dire le mal moral, avec une partie de la tonalité affective de tous nos présents qui ne se recouvre pas même avec le mal physique. Il faut se refuser à concéder à Nietzsche que la souffrance, dans son sens plénier, soit en vérité la Mère de l'Être.

L'éclaircissement qu'en fournit Henry est-il suffisant ? Sa thèse, toujours soutenue à l'identique, est bien que la souffrance, en principe et d'abord innocente, passe dans le mal moral lorsque la subjectivité, « au lieu de s'abandonner à l'œuvre de la vie en elle et à sa lente conversion dans la joie, n'en pouvant plus de se supporter ellemême, entreprend de se tourner contre soi, de se défaire de soi ». ${ }^{2}$ Le Je, dans son souci égoïque (ou déjà égoïste ?) de soi et dans sa profonde ignorance des tréfonds trop riches de son Soi divin, perd patience et décide de disposer d'un pouvoir qui en vérité surpasse de beaucoup les pouvoirs dont il a déjà conquis la possession. L'Archi-Fils, au contraire, obéit toujours, jamais ne perd patience, jamais ne permet que ne le domine ce minimum de souci de soi inclus dans le simple usage du mot «e ».

\section{5/ La phénoménologie radicale comme gnosticisme}

Mais alors qu'est en définitive le Soi, ce Soi singulier, qui s'appelle aussi le Premier Vivant ou le Fils unique de la Vie ? Et qui est le Soi de chaque Je, fini ou infini ?

\footnotetext{
${ }^{1}$ Cf. ibid., p. 88 : « La souffrance n'est pas dans le monde, mais le monde est dans la souffrance ».

${ }^{2}$ Ibid., p. 93.
} 
Cet Ipse que la Vie ne peut pas manquer d'engendrer constamment n'est que

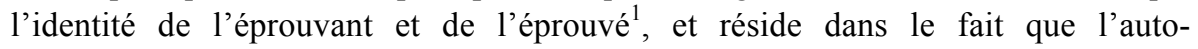
phénoménalisation de la phénoménalisation n'est pas a-subjective mais relève du savoir d'un Moi (d'un Je) archi-primordial. Mais le problème se pose immédiatement : si, dans mon expérience immanente de moi-même, mon Soi a bien la structure d'un Je, il y a deux sujets - et non pas seulement mon Soi et mon Moi, étant donné que mon Soi est forcément un Moi plus Moi que Moi-même. ${ }^{2}$ Du reste, il semble bien qu'il faille ménager un espace à l'épreuve de soi dans la subjectivité finie, un lieu souvent investi par les contemporaine de Henry voire par Henry luimême, et lié au fait que l'on est « rivé à soi, [...] écrasé contre soi, chargé de soi et se supportant soi-même dans sa corporéité pathétique et dans sa chair indéchirable ». ${ }^{3}$ Cela équivaut à exiger, pour chacun de ces sujets finis souffrants, un soi lui-même fini bien différent de l'Esprit ou du Logos, un soi que l'on a constitué au fur et à mesure que l'on a vécu, et non plus ce Soi qui, à la façon de la memoria dei d'Augustin, jaillit secrètement en nous vers la vie éternelle (ou qui est cette vie éternelle elle-même, dira Henry). Personne n'est rivé au ciel...

Dès lors s'agirait-il d'appréhender notre soi comme étant pour ainsi dire composé de deux couches - l'une relevant de la Vie absolue et l'autre de l'existence finie et pécheresse - et de comprendre la confusion qui s'y installe à l'aide du péché. Je ne supporte guère de continuer d'être moi-même justement parce que je reste tout à fait incapable de découvrir, au Fond de mon moi, la présence, l'illumination ou l'image de l'Esprit (l'une quelconque de ces trois choses suffirait évidemment à me redonner des forces qu'aucun événement ne pourrait renverser en pure détresse et en désespoir). Une chair éclairée par l'icône brûlante de la Trinité saute plutôt la barrière de la mort, au lieu de s'y soumettre volontiers en cherchant à s'y libérer de soi-même. L'amour qui me rédime fortifie ma chair à l'infini, jusqu'au sacrifice absolu de moimême, déjà accueilli dans les Demeures de la Trinité.

Comme il arrive souvent aux limites extrêmes des affirmations henryennes, nous trouvons ici des expressions proprement vertigineuses suivies de près par d'autres qui affaiblissent ce vertige. À la première classe appartiennent sans doute celles-ci : «Ma Chair n'est pas ma chair mais celle du Christ... Impossible de toucher une

\footnotetext{
${ }^{1}$ Cf. ibid., p. 105.

2 J'ai déjà employé cette formule, dont l'origine est relativement indépendante de Henry et qui à l'évidence développe une partie des intuitions augustiniennes, dans mes essais sur la philosophie de la mystique (De estética y mística, Salamanca 2007). Elle signale le fait, inattendu dans la philosophie transcendantale classique et même dans la phénoménologie "historique », que la subjectivité radicale se vit soi-même comme contemplée au moins par le regard divin, plus intérieur à mon immanence que mon « je ». La distance qui me sépare de Henry est que, dans ma perspective, il ne faut pas dire que mon Soi est le Christ ou l'Esprit : il est en vérité une image ou, mieux, une trace de la Trinité, car la véritable imago et similitudo trinitatis est le rapport d'amour - dilectio et benevolentia - intersubjectif.

${ }^{3}$ Ibid., p. 106.
} 
chair sans toucher la chair de Christ $»{ }^{1}$ Mais cette identité foncière se trouve atténuée dès lors que l'on reconnaît à chacun son Soi transcendantal individuel, « qui est à jamais le sien ». La vérité semble plus complètement exprimée dans la théorie de ces deux couches que j'ai esquissée, car «être un Soi n'est possible que dans le Christ », de telle façon que notre condition consiste en ce qu'Il « soit en nous avant que nous soyons à nous-mêmes », et cela dans un sens parfaitement littéral. ${ }^{2}$ Ainsi, lorsque le phénoménologue radical s'abandonne à son verbe foudroyant, il pose que cette chair qui est la mienne ne m'appartient pas en toute rigueur, parce que « j'ai été apporté en elle dans la venue en soi de la vie révélée à soi dans l'Ipséité de Premier Soi. C'est dans la chair de ce Premier Soi que j'ai pris chair, que je suis venu dans la condition d'un homme ${ }^{3}{ }^{3}$

Cette thèse se formule dans le cadre d'une discussion sur la valeur philosophique de la gnose, mais n'est pas forcée par le contexte : elle éclaire parfaitement le caractère non événementiel de notre naissance individuelle (il faudrait placer « individuelle » entre guillemets, parce qu'il s'ensuit effectivement que « le Soi vivant n'est en quelque sorte rien $»){ }^{4}$ Mais la naissance du Christ, son Incarnation même, perdent aussi leur caractère événementiel, et la Chair du Christ accompagne son éternel engendrement. La kénose serait le s'auto-vider du Père, comme au temps des discussions patripassiennes et sabelliennes propres à l'Église des premiers siècles, ${ }^{5}$ bien que Henry attribue plutôt sa thèse à Irénée - elle serait tout simplement le cogito des chrétiens, une autre façon, contemporaine, d'exprimer phénoménologiquement la manière dont l'hypostase du Christ unifie ses deux natures. ${ }^{6}$

Si Henry a pu identifier sa phénoménologie radicale au christianisme, c'est en en soustrayant tout ce qui à ses yeux y relèverait du métaphorique et de l'ordre du « comme si ». Mais l'hyperréalisme chrétien ne comporte pas la suppression de l'analogie et du sens foncier de la transcendance. Surtout, il aurait fallu ne pas saisir toujours l'image comme telle dans le sens du Dehors et du Monde. ${ }^{7}$ C'est tout autre

\footnotetext{
${ }^{1}$ Ibid., p. 127.

${ }^{2}$ Toutes ces citations sont tirées des pages 126-128 de Phénoménologie de la vie, t. IV. Cet essai fut d'abord publié dans la revue Communio, et l'on peut supposer que Henry n'y aura pas souhaité s'abandonner aux audaces expressives qui le rapprochent tant d'Eckhart. Un autre texte parle de l'« intériorité phénoménologique réciproque» (ibid., p. 142; la p. 154 corrigera : «non réciproque ») entre l'homme et le Logos, laquelle n'est que la façon, en phénoménologie radicale, d'exprimer la proposition de deux couches dans le Soi « individuel à jamais ».

${ }^{3}$ Ibid., p. 138. Il faut ajouter qu'aucune différence entre l'âme et le corps n'est admissible.

${ }^{4}$ Ibid. Henry ose finir son texte ainsi : «Le christianisme n'a pas à se soustraire au soupçon de gnosticisme » (ER 143).

5 «Pour moi, Dieu est pathétique », déclara Henry au théâtre de l'Odéon en 1999. La phrase suivante rappelle que « Dionysos, c'est la souffrance et la joie» (ibid., p. 214).

${ }^{6}$ Cf. ibid., p. 151-153.

${ }^{7}$ Cf. ibid., p. 180. Inutile alors de se restreindre à ne parler que de « quasi-identité de l'essence de l'homme et celle de Dieu » (ibid., p. 186).
} 
chose d'affirmer que 1'Esprit de Dieu habite dans l'homme et d'affirmer que tel est également le cas du Christ. ${ }^{1}$

\section{6/ Intersubjectivité et altérité}

Je soutiens que, de même que la vraie icône de Dieu ne se trouve pas dans l'isolement d'un Je (ni même dans un dialogue intime ou un soliloque avec son Soi) mais dans l'amour (dilectio vel benevolentia) donné et reçu dans sa fécondité immédiate (tókos en kalôi, selon la parfaite expression du Banquet), de même la phénoménologie (absolument) radicale rencontre son problème central dans l'événementiel personnel, dans le rapport inter-subjectif.

Le mérite indiscutable de Henry, qui lui a fait gagner, et pour toujours, une place d'honneur dans la philosophie du $\mathrm{XX}^{\mathrm{e}}$ siècle, a été de mener à bout et sans concession le programme strict d'une phénoménologie (absolument) radicale. À nous ses élèves, il reste alors à faire état de notre situation et à constater, avec la même radicalité que notre inspirateur, que nous nous trouvons, avec cette ontologie phénoménologique conduite avec tant cohérence, très au-delà de Heidegger, Sartre ou Merleau-Ponty.

Je souligne le point central de ma position : l'événementiel personnel diffère toto coelo de je ne sais quel pouvoir chosique, mondain, du réel auquel notre subjectivité serait religata, comme d'autres ont cru pouvoir le promouvoir (justement avant d'avoir sous les yeux la pleine réalisation du programme impossible de cette phénoménologie absolument radicale). La réduction de l'Un à la seule Vie qui existe, à la Vie absolue, comprise comme auto-révélation éternelle historiale, autoengendrant éternellement en elle l'Archi-Ipséité de la Chair divine, outrepasse le programme néoplatonicien et, comme le disait saint Jean de la Croix, nous permet d'«atteindre le gibier » que toute cette antique tradition n'avait considéré comme atteignable que dans l'excessus mystique et, peut-être, après la mort d'une existence radicalement purifiée au sens gnostique de la kátharsis — et atteignable, précisément, comme l'auto-lumière qui m'éclaire du dedans et illumine tout le reste du réel dans la même immanence (parfois impuissamment rejetée et interprétée comme pur Monde mort). Ce n'est plus l'intellectus-Vernunft du Cardinal de Cusa; ce n'est plus la docta ignorantia ni la philosophia, mais bien la sophia, la theosophia, la Wissenschaft plutôt que la Wissenschaftslehre fichtéenne. Ici le Savoir n'est plus l'Image de l'Amour, mais l'Amour lui-même, bien que pris tout d'abord dans le brouillard d'une chair qui ne reconnaît pas la divinité foncière de son Soi. La réduction phénoménologique réussira par la suite à dissiper ces nuages, et la veille définitive montrera la nullité, le néant de tout Monde, de tout Temps, de tout Individu isolé. La Chair divine nous rallie tous, nous soutient tous dans notre épreuve abso-

\footnotetext{
${ }^{1}$ Cf. ibid., p. 169.
} 
lue-finie de la Vie (et finie parce que, en dernière instance, incapable du Savoir parfait — parinirvâna). Il y a déjà plus que l'attingere inattingibile quod est inattingibile, selon les mots de l'Idiot de sapientia.

La seule possibilité pour une phénoménologie quasi-radicale, ou pour une philosophie première quasi-phénoménologique, est la théo-logie, et non la théo-sophie. C'est la structure de l'amour personnel et de la liberté inter-subjectivement exercée qui décide du sort final de la philosophie première. L'Un n'est ni caché ni possédé, mais Tri-Un, peut-être, et imageable (imaginable?), sans doute. Il n'y a qu'une ontologie pluraliste sans dissémination, phénoménologique mais foncièrement éthico-religieuse, qui puisse satisfaire au rang historial et vraiment événementiel des phénomènes qui ne sont pas que des expériences d'auto-accroissement et de pathos auto-affectionnel, mais aussi des sauts de liberté et, surtout, des événements qui traumatisent et dramatisent la vie finie — jusqu'à la tragédie du mal, du désespoir, du péché, du nihilisme, mais aussi jusqu'au ciel des formes inouïes de communauté et d'amour et d'expériences mystiques à plusieurs sujets.

L'ontologie dans les extrêmes de l'effort phénoménologique suit beaucoup plus les voies de Levinas, de Nabert et, surtout, de Kierkegaard, que celles de Nietzsche ou de Heidegger; elle descend de Socrate plutôt que d'Épicure ou de Zénon. Les événements qu'elle a à reconnaître comme absolument signifiants au fil des jours de la vie finie sont ceux de la mort, de la compassion et du pardon, et la phénoménologie radicale en a frayé le chemin en montrant qu'il n'était pas possible de saisir phénoménologiquement, jusqu'au bout, le sens de ces événements. C'est pour cela que je reste l'élève dévoué de Michel Henry, en même temps que je m'éloigne du contenu factice de son enseignement dans la conviction que c'est l'esprit du même radicalisme qui me pousse dans ma propre direction.

«Chacun s'aime dans un "autre" qui ... ne lui est jamais extérieur mais intérieur et consubstantiel $»^{1}$. Cette assertion cadre avec notre compréhension du rapport complexe entre le Logos-Chair, le Soi et le Je. Les deux premiers membres de cette série s'identifient : mon « soi » singulier se compose d'une couche essentielle, fondamentale, divine, et d'une autre qui m'appartient en propre comme un produit de mon auto-constitution (inévitable depuis que je me suis approprié de moi-même, c'est-à-dire du Soi qui donne à vivre à tout homme). ${ }^{2}$ Mon existence à moi laisse se sédimenter une mémoire et des habitudes transcendantales qui dépendent de mes expériences, de mes événements et de mes sauts de liberté, aussi bien que de cet

\footnotetext{
${ }^{1}$ Ibid., p. 157. Dans le bel article «Le Berger et ses brebis », Henry s'écrie : « Je ne suis pas ma propre chair. Ma chair, ma chair vivante est celle du Christ» (ibid., p. 168). Le problème évident est que ma chair pèche, que le mal moral s'intègre dans la chair de ma vie et dans mon « soi »...

${ }^{2}$ Dans ibid., p. 159, Henry soutient bien que « le Soi primordial... contient la multiplicité potentielle et indéfinie de tous les moi possibles », tandis que « la relation de chaque Soi transcendantal à la vie absolue [est] le lien religieux (religio) » (c'est Henry qui souligne).
} 
autre élément indéniable, mais peut-être inexpliqué dans la théorie de la phénoménologie radicale, qui est le caractère tout-à-fait individuel de mon corps subjectif (masculin ou féminin, agile ou maladroit, sain et jeune ou abîmé par l'âge, la maladie et l'usage). Or ce qui importe primordialement, autant pour l'éthique que pour la religion elle-même, n'est pas seulement mon amour adressé à moi-même et véhiculé plus ou moins secrètement par le Logos, mais l'amour pour autrui et l'amour d'autrui.

C'est une vérité importante d'affirmer que toute communauté, puisqu'elle est communauté dans la vie, est invisible; mais c'est une autre vérité importante de rappeler que, comme le disent les vers de saint Jean de la Croix, mira que la dolencia / de amor, que no se cura / sino con la presencia y la figura. ${ }^{1}$ L'espace affectif (toujours espace de la distance et la séparation), conditionné par la Terre, relève aussi d'une dimension éthique et religieuse inestimable. L'amour se comble aussi de la distance, mais sa plénitude véritable exige le voyage auprès de la chair souffrante, de la chair aimante, du lieu de l'engagement.

La thèse capitale qui voit dans la Vie «l'être avec comme tel » a absolument besoin d'une preuve concernant le fondement de la communauté humaine. Pourquoi la multiplication de la Chair en tant de chairs ? Pourquoi la multiplication du Vivant en tant de vivants? Nous questionnons sur le principium individuationis personarum, parce que l'on ne voit, d'abord, ni la nécessité ni, bien pire, la possibilité de tant d'individus finis différents.

Ne pas comprendre la nécessité de la création d'une personne finie (ou d'un grand nombre de telles personnes) permet sans doute de préserver pour la Vie la qualité foncière de l'Amour. Mais comment ne pas vouloir comprendre (au moins comprendre !) comment le Vivant peut engendrer des vivants dès qu'il le veut ? $^{2}$ Or la tentative de le faire au fil de l'idée selon laquelle mon corps, par exemple, peut «achever ce qui n'était pas encore achevé dans le Christ», reste insuffisante, à moins que l'on ne saisisse événementiellement le Christ. Mais c'est justement cela qui est défendu par l'interprétation " gnostique » de l'Évangile explicitement avancée par Henry, comme on l'a observé. La finitude de l'individu peut bien se dire dans des termes très proches de ceux de Kierkegaard : mon rapport à moi-même, je l'ai reçu, il a été posé par un autre (un Autre qui y reste comme couche fondamen-

\footnotetext{
${ }^{1}$ Canción 11 du Cantique spirituel: « Vois que le mal d'amour n'est pas guéri qu'avec la présence et la figure ».

${ }^{2}$ Cf. Phénoménologie de la vie, t. IV, op. cit., p. 174 : « La vie fait sa place à tout vivant concevable. Ainsi contient-il a priori dans son essence la multitude indéfinie de tous ceux qu'elle peut appeler à la vie». Mais la question est celle de cette concevabilité ! L'unicité de la vie exige (et suppose) un vivant unique. Comment comprendre un individu fini ? Comment comprendre une multitude indéfinie d'individus finis?
} 
tale du Soi-même fini) $;^{1}$ mais le problème est de concevoir cette position ou cette naissance aussi bien du côté du Christ que du côté de l'homme.

La seule description possible de la finitude individuelle est l'oubli: «C'est seulement dans l'Oubli de son Corps que le moi se trouve en possession de tous ses pouvoirs », dans l'Oubli de l'Immémorial, dans l'Oubli du Passé de l'Historial. À chaque présent nous observons ce même phénomène extraordinaire : que la vie «se sait sans se savoir » et que la phénoménologie radicale, anticipée par le christianisme de la théologie johannique, s'attarde sur la scène de la philosophie jusqu'à la fin du XX $X^{\mathrm{e}}$ siècle. Or l'Oubli, ce double oubli du Passé et du Présent vivant, a besoin d'un fondement: d'un péché originel ou d'une création à l'image et à la ressemblance de Dieu. Le fait est l'Oubli ; la théorie est... la phénoménologie radicale. À Michel Henry de reconnaître qu'il «n'est pas au pouvoir de la phénoménologie d'expliquer » le processus de venue dans la vie d'un vivant fini. ${ }^{2}$ Mais la phénoménologie radicale pourrait plutôt renoncer à la saisie du premier principe absolu qu'à celle d'un moment déjà si dérivé du procès éternel de la Vie ! Ainsi la philosophie première se doit de reculer à une docta ignorantia très théologique, mais pas gnostique.

\footnotetext{
${ }^{1}$ Encore une citation, parmi bien d'autres identiques : «Le soi de la vie est la possibilité intérieure permanente de mon soi » (ibid., p. 224).

${ }^{2}$ Ibid.
} 